\title{
Kalman Filtering Technique for Video Denoising Method
}

\author{
Lakshmanan.S \\ PG.Scholar
}

\author{
Mythili.C \\ Asst.Professor ( ECE Dept) \\ University College of Engineering, Nagercoil
}

\author{
V. Kavitha. \\ Director
}

\begin{abstract}
Digital Cameras which capture images and videos are directly in digital form .Digital Images or Videos are often corrupted by impulse noises. It is caused by disturbances and corrupted in the video signal. So the image processing scheme should be one of the important part in any vision application permitting to suppress noise and improve the image performances. This demands to have number of filtering schemes are introduced such as fuzzy and nonfuzzy and linear and non-linear are used. In this paper, propose Kalman filter is used to remove the impulse noise. Kalman filter is the best and efficient filters in the sense of minimizing mean square error (MSE) and high PSNR (peak signal to noise ratio) between the original video signal and recovered video signal.
\end{abstract}

\section{Keywords}

Cameras, Cameras ISO, Colored Image, Impulse Noise, Kalman filter, Recursive algorithms.

\section{INTRODUCTION}

Images \&Videos belong to the most important because more information collected from both sequences. The images are likely to be corrupted by noise due to bad acquisition ,transmission or recording and also depends upon the cameras parameters. Such degradation negatively influences the performances of many image processing techniques and a preprocessing module to filter the images is often required. Impulse noise is modeled as bit errors in the signal values during transmitting the images or video sequences over noisy data links. The high sensitivity image quality of a given camera may depend greatly on the quality of the algorithm used for noise reduction .Different classes of filters have been proposed for removing noise from gray scale \& color image.In this paper a recursive and feedback control based Kalman filter is proposed. This filter is working with different successive filtering steps a very good tradeoff between detail preservation \& noise removal is obtained .In each step noisy pixels are detected by the help of fuzzy rules.

In Section1 proposes introduction of propose impulse noise filtering techniques. In Section2 proposes Related works for some impulse noise filtering techniques. In Section 3 proposes Methodologies of propose filtering techniques. Finally, the Experimental results of propose filtering techniques.

\section{RELATED WORKS}

Most algorithms for converting image sensor data to an image, whether in-camera or on a computer, involve some form of noise reduction. There are many procedures for this, but all attempt to determine whether the actual difference in pixel values constitute noise or real photographic detail. So there is often a trade-off made between noise removal and preservation of fine, low-contrast detail that may have characteristics similar to noise. Many cameras have setting to control the aggressiveness in camera noise reduction. The high sensitivity image quality of a camera may depend greatly on the quality of the algorithm used for noise reduction. Since noise levels increases as ISO sensitivity is increased, most camera manufactures increase the noise reduction [1]. They are classified into several categories depending on specific for Gaussian noise and impulse noise removal have poor performance against impulsive noise.

Linear filtering techniques have been used in many image processing applications. Linear filter is not suitable to tend effectively remove heavy tailed noise, and perform poorly in the presence of signal-dependent noise. To overcome the drawback of linear filter introduced a non linear filtering technique [2].The drawback of non-linear filter should be carefully selected depending on both the characteristics of video and its noise disturbances characteistics.

In recent progress, removal of both Impulse and Gaussian noise in color images introduced fuzzy logic allows different possibilities for developing new image noise reduction methods and also used fuzzy logic membership functions. For dealing with the impulse noise, an algorithm is developed to search for a set of uncorrupted pixels in the neighborhood of the pixel of interest. A kalman filter consisting is proposed to cancel out the impulse noise [3].These fuzzy filters are able to outperform sequences-order filter schemes. Therefore, this paper presents a new technique for filtering impulse noise by a kalman filter. We present a filter for the removal of random impulse noise in color image sequences, in which each of the color components is filtered separately based on fuzzy rules. To preserve the details, the noise is removed by three successive filtering steps. To benefit as much as possible from the spatial and temporal information available in the sequence. The experiments show that the proposed method outperforms other filtering methods and in terms of objective quality measures such as the MSE, PSNR.

\section{METHODOLOGY}

In this work, a new Kalman filter introduced for the removal of random impulse noise in different steps .In each successive filtering step, noisy pixels are detected and filtered with the help of recursive algorithms with feedback control [4].

Step I: In the first step is detect noisy pixel and noiseless pixels is based on the recursive and spatial- temporal information.

Step II: In the second step is based on color information. An image pixel component can be seen as noisy if there is no similarity to its (spatio-temporal) neighbors in the given color, while there is in the other color bands. 
Step III: Finally, removes the remaining noise and refines the result by using as well as Spatial-temporal and color information.

\subsection{KALMAN FILTERING SYSTEMS}

Zhai Ming and Fushan introduced kalman filtering using a noise reduction techniques. The kalman filter was a linear estimator based on MMSE(Mean Square Error)[5,6,7] for the sake of less amount of computation and better performance it had been widely used since it was introduced. The Kalman filter is a computationally digital signal processing based filter. It can give estimates of past, present and future states of a system even when the underlying model is imprecise or unknown. For more on the kalman filter there are many good references to explain it..The two most important aspects of the Kalman algorithm are the systematic model and the noisy models. The kalman filter are based on linear systems discretized in the time domain. They are modeled on a Markov chain built on linear operators perturbed by Gaussian noise Suppose Kalman Filter is used for linear noise system model need for two obvious requirements come to mind. First, we want the average value of our state estimate to be equal to the average value of the true state. That is, we don't want our estimate to be bias done way or another. Mathematically, the expected value of the estimate should be equal to the expected value of the state. Second, we want a state estimate that varies from the true state as little as possible. That is, not only do we want the average of the state estimate to be equal to the average of the true state, but we also want an estimator that results in the smallest possible variation of the state estimate. But the Kalman filter solution does not apply unless we can satisfy certain assumptions about the noise that affects our system. The Kalman filter estimates a process by using a form of feedback control: First the process state is estimated using previous state variables and the defined linear system equations, then a feedback is obtained from the measurements. Therefore, the operation of the Kalman filter can be divided into two parts; the prediction stage (time update equations) and the correction stage (measurement update equations). The time update equations are responsible for projecting forward(in time) the current state and error covariance estimates to obtain the a prior state estimates. The measurement update equations are responsible for the feedback, by incorporating a new measurements into the a prior estimate to obtain an improve a posteriori estimate .For instance, different cameras are used in various applications and wireless video communications the motion dynamics are such that changes in the direction and speed of camera motions are important likely jitter. Therefore, the process noise cannot be restricted to very low values to ensure that the Kalman filter can adjust to change in the motion dynamics, and the measurement noise variance has to be set to a moderate value that will ensure that appropriate stabilization is performed, and at the same time intentional camera movements are followed closed. The Kalman filtering noise removal block diagram as shown in fig1

\subsubsection{Filtering Algorithm}

Consider random process $\mathrm{X}(\mathrm{n})$ and $\mathrm{Y}(\mathrm{n})$ such that,

$$
\begin{gathered}
X_{n+1}=A_{n} X_{n}+W_{n} \\
Y_{n}=A_{n} X_{n}+N_{n}
\end{gathered}
$$

Where $\mathrm{W}_{\mathrm{n}}$ and $\mathrm{N}_{\mathrm{n}}$ are independent Gaussian random process and independent of $\mathrm{X}$, clearly, $\mathrm{X}_{\mathrm{n}}$ is a Markov process which together with the observations $Y_{n}$ forms a Hidden Markov process. The problem of obtaining the best estimate of $\mathrm{X}$ from the observations $\mathrm{Y}$ require one to estimate the conditional probabilities $\mathrm{P}\left(\mathrm{X}_{\mathrm{n}} / \mathrm{Y}^{\mathrm{n}}\right)$. This is a accomplished in a computationaly efficient manner by the forward recursion algorithm. In order to apply to video signals, we put the previous two equations in the form shown in equations (3) and (4) and we define the parameters as follows, $X_{n}$ : actual value of pixel in current frame. $X_{n-1}$ :actual value of pixel in previous frame. $\mathrm{W}_{\mathrm{n}}$ : Gaussian random process represents error in motion estimation using blockmatching, $\mathrm{Y}_{\mathrm{n}}$ : noisy observation of pixel in current frame. $\mathrm{N}_{\mathrm{n}}$ : additive Gaussian noise to be removed. This model makes the Kalman filter assumptions Gaussian independent process hold true.

$$
\begin{aligned}
& X_{n}=X_{n-1}+W_{n} \\
& Y_{n}=X_{n}+N_{n}
\end{aligned}
$$

Clearly $\left(\mathrm{X}_{\mathrm{n}} / \mathrm{Y}^{\mathrm{n}}\right.$ and $\mathrm{X}_{\mathrm{n}} / \mathrm{Y}^{\mathrm{n}-1}$ are Gaussian random variables.Denote the mean and variance of these random variables by $\alpha_{n}^{(0)}, \alpha_{n}^{(1)}, \beta_{n}^{(0)}, \beta_{n}^{(1)}$ respectively. Then the update equations become,

$$
\begin{gathered}
\alpha_{\mathrm{n}}{ }^{(0)}=\beta_{\mathrm{n}}{ }^{(0)}+\frac{\beta_{\mathrm{n}}{ }^{(1)}}{\beta_{\mathrm{n}}{ }^{(1)}+\sigma_{\mathrm{n}}{ }^{2}}\left(\mathrm{Y}_{\mathrm{n}}-\beta_{\mathrm{n}}{ }^{(0)}\right) \\
\alpha_{\mathrm{n}}{ }^{(1)}=\beta_{\mathrm{n}}{ }^{(1)}+\frac{\beta_{\mathrm{n}}{ }^{(1)}}{\beta_{\mathrm{n}}{ }^{(1)}+\sigma_{\mathrm{n}}{ }^{2}} \\
\beta_{\mathrm{n}+1}{ }^{(0)}=\alpha_{\mathrm{n}}{ }^{(0)} \\
\beta_{\mathrm{n}+1}{ }^{(1)}=\alpha_{\mathrm{n}}{ }^{(1)}+\sigma_{\mathrm{w}}{ }^{2}
\end{gathered}
$$

Where $\sigma_{\mathrm{n}}{ }^{2}$ is the noise variance and $\sigma_{\mathrm{w}}{ }^{2}$ is the variance of the process $\mathrm{W}$. We estimate the variance of $\mathrm{W}$ as follows. We first estimate the mean of the pixel values in surrounding block in the present frame $\mu_{\mathrm{n}}$ and matching block in the previous frame $\mu_{\mathrm{n}-1}$. Then, $\sigma_{\mathrm{w}}{ }^{2}$ is estimated as $\sigma_{\mathrm{w}}{ }^{2}=\left(\mu_{\mathrm{n}}-\mu_{\mathrm{n}-}\right.$ $\left.{ }_{1}\right)^{2}$.This is a reasonable estimate of $\sigma_{\mathrm{w}}{ }^{2}$ since w tries to capture the difference between pixel values at successive frames. Also, we initialize $\beta_{0}{ }^{(0)}$ and $\beta_{0}{ }^{(1)}$ to the mean and variance of $\mathrm{X}_{0}$ which we estimate from the first frame.

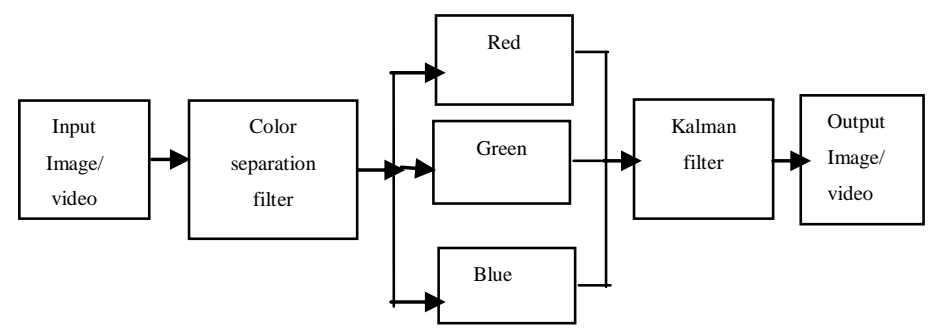

Fig1.Kalman Filtering Noise Removal Process 
An input image and videos are captured by different cameras. These images may be still photographs. An image is digitized directly or to convert which can be stored in a computer memory or storage media. Segmentation is a process that partitions an image into regions. Suppose the objective is to segment objects of a specific color range in an RGB image. Image noise is generally regarded as an undesirable by-product of image capture. The impulse noise is caused by sharp, sudden disturbances in the image signal. Noise filtering can be viewed as removing the noise from corrupted image and smoothen it so that the filtered image can be viewed.. To preserve the details as much as possible, the noise is removed by Kalman filtering techniques based on recursive, spatial and temporal information. To benefit as much as possible from the spatial and temporal information available in the sequence. The experiments show that the proposed methods outperforms in terms of MSE,PSNR[5]. Finally, the proposed filter combines very good detail of image edge preservation to very good noise removal and clearly reduces the mean squared error compared with other filters.

\section{EXPERIMENATL RESULTS}

The performance of the proposed method, we will use the Mean Square Error (MSE) measures of similarity between a filtered image and the original image. A color image consisting of an $\mathrm{M} \mathrm{x}$ $\mathrm{N} \times 3$ array of pixels at locations $(\mathrm{x}, \mathrm{y})$. The performance measurements of videos as shown in Table1 and Table2.

$$
\operatorname{MSE}(f, I)=\sum_{z=1} \sum_{x=1} \sum_{y=1}^{N}[I(x, y, z)-f(x, y, z)]^{2}
$$

$$
3 \times \mathrm{N} \times \mathrm{M}
$$

Where $\mathrm{I}$ is the original image, $\mathrm{f}$ is the noiseless image or filtered image of size $\mathrm{N} \times \mathrm{M}$.

Another similarity measures is PSNR (peak signal to noise ratio) which is related to MSE as in the following:

$$
\operatorname{PSNR}(\mathrm{f}, \mathrm{I})=10 \log _{10} \frac{1}{\operatorname{MSE}(\mathrm{f}, \mathrm{I})}
$$

Table 1: Performance measurements of Video1

\begin{tabular}{|c|c|c|}
\hline \multirow{2}{*}{$\begin{array}{c}\text { Noise } \\
\text { Density }\end{array}$} & KSNR(db) & MSE \\
\cline { 2 - 3 } & & \\
\hline \multirow{2}{*}{0.1} & 25.7654 & 172.4017 \\
\hline 0.3 & 26.8294 & 134.9407 \\
\hline & & \\
\hline 0.5 & 26.49 & 132.94 \\
\hline
\end{tabular}

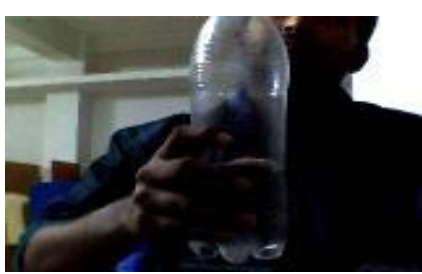

a. Input Video sequences

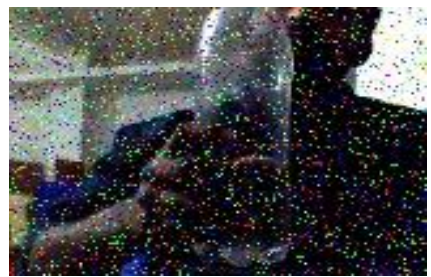

b. Noisy video $0.1 \%$

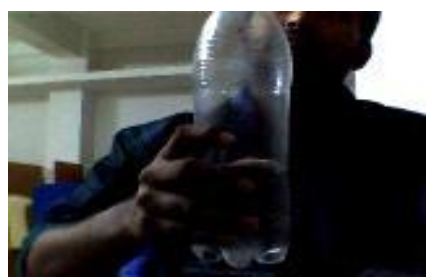

c. filtered output

Fig 2. Performances of Video I

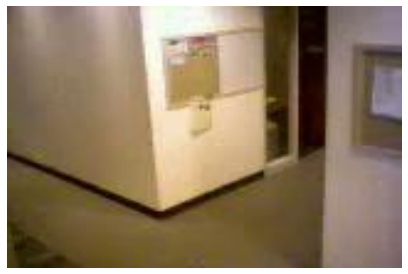

a.Input Video sequences

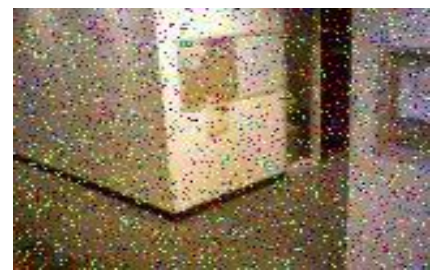

b .Noisy Video $(0.1 \%)$

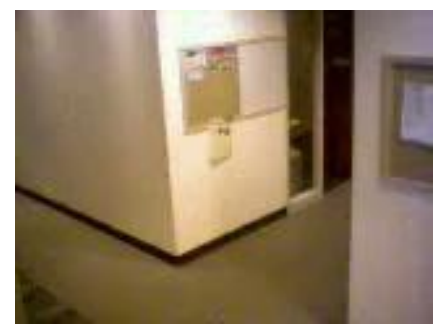

c. filtered video

Fig 3.Performances of Video2

Table 2: Comparison of Performance (Video2)

\begin{tabular}{|c|c|c|}
\hline \multirow{2}{*}{ Noise Density } & \multicolumn{2}{|c|}{ Kalman Filter } \\
\cline { 2 - 3 } 0.1 & PSNR(db) & MSE \\
\hline \multirow{2}{*}{0.3} & 26.0257 & 142.22 \\
\hline & 26.3096 & 159 \\
\hline 0.5 & 26.85 & 134.17 \\
\hline
\end{tabular}




\section{CONCLUSION}

In this paper we have presented a new filtering framework for color videos / Images corrupted with random valued impulse noise induced from different cameras. By using Kalman filtering noise removal algorithms noise is removed step by step. The experiments showed that the proposed method outperforms in terms of MSE,PSNR values are varied depends upon the parameters of different cameras videos performances of using different application. Our future research will be focused on the improvement of videos performances in PSNR and reduced MSE and other performances .

\section{REFERENCES}

[1] Francisco Gallegos, volodymyr Ponamaryov, " Order Statistics - Fuzzy Approach in processing of Multichannel Images and Video Sequences " National polytechnic Institute of Mexico .

[2] A.Ben Hamza and Hamid Krim, “ Image Denoising : A Non linear Robust Statistical Approach " IEEE Transactions on signal processing, vol 49,No.12, December 2001 pp 3043-3045.

[3] Andrzej Lesniak, Tomasz Danek " Application of Kalman filter to noise reduction in multichannel data" Vol 17/18 ,2009

[4] Scarp Erturk,"Real Time Digital Image Stabilization Using Kalman Filters,"Real Time Imaging Vol-8,p.no 317-328.2002

[5]Tom Melange,Mike Nachtegael,'Fuzzy Random Impulse Noise Removal From Color Image Sequences", IEEE transactions on Image processing, vol 20,No.4 April 2011

[6] Carl Steven Rapp,'Image processing and Image Enhancement",Texas,1996.

[7] R.Vorobel,"Contrast Enhancement of Remotely-Sensed Images,"Sept 1996,pp 472-475

[8] Bravn,R.G.\& Huwang,P.Y.C.(1992) Introduction to Random Signals and Applied Kalman filtering $\left(2^{\text {nd }} e d n\right)$. New York;Jam Wiley\&Sons.

\section{AUTHOR'S PROFILE}

M.S.Lakshmanan received her B.E degree in Electronics and Communication Engineering 2006 from Anna University, Chennai Presently he is working as Lecturer in the Department of ECE Sun Institute of Technology,Erachakulam in Kanyakumari District. Currently he is pursuing his ME degree in Applied Electronics in University College of Engineering,Anna University of Technology ,Nagercoil.

Mrs.C.Mythili received her BE degree in Electrical and Electronics Engineering 2001 from MS university and ME degree in Applied Electronics in2004 from Anna university Chennai.She is university fourth rank holder in PG.Presently she is working as Asst.Prof in the Department of ECE university college of Engineering Annauniversity of Technology Nagercoil.Currently she is pursuing her Ph.D from Anna university Tirunelveli.Her research area includes Image Segmentation,Image Enhancement and Image Compression

Dr.V.Kavitha obtained her B.E degree in Computer Science and Engineering in 1996 from MS University and ME degree in Computer Science and Engineering in 2000 from Madurai kamaraj University.She is University Rank Holder in UG and Gold Medalist in PG.She received PhD degree in Computer Science and Engineering from Anna University Chennai in the year 2009. Right from 1996,she is in the Department of Computer Science and Engineering under various designations.Presently she is working as Asst.Prof in the Department of CSE at Anna university Tirunelveli.In addition she is the Director In-charge of University College of Engineering ,Nagercoil.Currently under the guidance twelve research scholars are pursuing $\mathrm{PhD}$ as full time and part time .She has published many papers in National and International journals in areas such as Network security,Mobile computing ,wireless network security,and cloud computing 